Angiogenic factors and preeclampsia: an early marker is needed

\title{
Holger Stepan
}

\section{Comment}

on : Bills et al. Failure to upregulate VEGF165b in maternal plasma is a first trimester predictive marker for preeclampsia

Correspondence:

Holger Stepan, MD

Department of Obstetrics, Leipzig University

Liebigstr. 20a

04103 Leipzig

Germany

phone $++49-341-9723595$

fax $\quad++49-341-9723599$

e-mail holger.stepan@medizin.uni-leipzig.de 


\section{Abstract}

Preeclampsia, a pregnancy complication characterized by hypertension and proteinuria, is still a major cause of neonatal and maternal mortality and acute and long-term morbidities for both, mother and neonate. There is mounting evidence that an imbalance between angiogenic factors such as VEGF or PIGF and factors inhibiting angiogenesis such as sFlt1 and sEng are closely related to the pathogenesis of preeclampsia. In the present issue of Clinical Science, Bills and coworkers report that VEGF $165 \mathrm{~b}$, an alternative splice variante of the VEGF-pre-mRNA, is upregulated in women with normal pregnancy and that this increase delayed or diminished in women who develop preeclampsia. Thus, this protein could serve (alone or in combination with other parameters) as a new marker for risk assessment in terms of preeclampsia. 
Preeclampsia, a serious disorder of pregnancy characterized by hypertension and proteinuria, complicates approximately $2-5 \%$ of all pregnancies. Although several risk factors for this condition are well recognized, including first pregnancy, low and high maternal age, multiple pregnancy, obesity, and preexisting diabetes or hypertension, the causes of preeclampsia remain unclear. As a consequence, no causal treatment is available and resolution of this syndrome occurs only after removal of the placenta out of the maternal body. Preeclampsia is still called the "disease of theories" and previous hypotheses included pathogenic factors like placental ischemia, inflammation and oxidative stress [1]. After a period of stagnation, progress has come into research of hypertensive pregnancy complications. Recent studies have suggested that changes in circulating angiogenic factors play a key role in the pathogenesis of preeclampsia. Increased expression of soluble fms-like tyrosine kinase-1 (sFlt1), associated with decreased PIGF and VEGF signaling, were the first abnormalities described. sFlt1 is a truncated splice variant of the membrane-bound VEGF receptor Flt1, also called VEGFR1. This protein consists of the extracellular ligand-binding domain without the transmembrane and intracellular signaling domains. Thus, sFlt1 has a biological activity as an antagonist to both VEGF and PIGF, binding them in the circulation and preventing interaction with their endogenous receptors. Placental expression of sFlt1 is increased in preeclampsia and is associated with a marked increase in maternal circulating sFlt1 [2]. Several investigators have confirmed that the increase in maternal circulating sFlt1 precedes the onset of clinical disease and is correlated with disease severity $[3,4]$. In parallel, PIGF concentrations are decreased in preeclamptic patients and this decrease also precedes the manifestation of the syndrome [5]. Recently, soluble endoglin (sEng), a cell-surface co-receptor for transforming growth factor (TGF)-ß1 and TGF-ß3 isoforms, has been identified as a novel antiangiogenic protein. Interestingly, it acts 
synergistically with sFlt1 to induce endothelial dysfunction and the simultaneous administration of both antiangiogenic proteins enhances the ability of sEng to induce microangiopathic anemia and thrombocytopenia [6]. A nested case-control study could demonstrate that pregnant women with preeclampsia have elevated sEng concentrations in circulation [7]. The increase of circulatory sEng is detectable about two to three months prior to the clinical manifestation of preeclampsia. Since the known sFlt1 increase is described as detectable five weeks before the onset of the disease, sEng could be an earlier marker for preeclampsia. Thus, it is our view today that preeclampsia is an antiangiogenic state and that the measurement of (anti)angiogenic factors in maternal blood is - due to the early alteration- effective for prediction and diagnosing.

In this issue of the Journal, Bills and co-workers [8] report that VEGF ${ }_{165} \mathrm{~b}$, an alternative splice variante of the VEGF-pre-mRNA, is upregulated in maternal blood from women with normal pregnancy. Interestingly, this increase of $V_{E G F_{165} b}$ is delayed or diminished in women who develop preeclampsia. Secondly, women with low VEGF ${ }_{165} \mathrm{~b}$ concentrations were characterized by higher sFIt1 and soluble endoglin levels, which indicates undoubtedly an increased risk for preeclampsia. The authors conclude that low VEGF $165 \mathrm{~b}$ may be also a risk factors and thus, that this protein could serve as an early marker. The observation of this paper points at the fact that the sequential change of an angiogenic/antiangiogenic factor may have more relevance than the measured absolute values. Thus, these findings are in line with data of Vatten [9] and Rana [10] who describe first observations that sequential changes of angiogenic factor differ in women destined to develop preeclampsia. For instance, a low increase of PIGF and a high increase of sFlt1 are strong predictors of 
preeclampsia. Moreover, women with early-onset preeclampsia show a pronounced increase of sFlt1 and sEng between first and second trimester [9, 10].

The report by Bills and co-workers [8] adds to the accumulating evidence linking alterations of the angiogenic-antiangiogenic balance and the pathogenesis of preeclampsia. The field of preeclampsia research is experiencing a well-deserved blossoming of new insights into the pathobiology of the "disease of theories". As a possible consequence, the research on angiogenic factors and the intervention on the angiogenic-antiangiogenic state will lead to much earlier diagnostic capacities and novel prophylactic treatments. However, the work of Bills and co-workers in this issue documents also that the problem of the angiogenic system in terms of preeclampsia is much more complex as once thought. VEGF ${ }_{165} \mathrm{~b}$ is described as a factor with antiangiogenic properties and thereby in line with sFlt1 and sEng. Thus, an increase of $\mathrm{VEGF}_{165} \mathrm{~b}$ or high levels rather than a delayed increase or low levels should be expected or "typical" for women at risk for preeclampsia. The finding that a new splice variante of the VEGF-pre-mRNA does not behave as expected underlines the complexity of the story. We learn that probably not a single angiogenic factor will serve as an early marker. Similar to the stepwise development of the screening for aneuploidies, a combination and algorithm of biochemical markers and other parameters (e.g. uterine Doppler) will allow risk estimation for preeclampsia in the future. 
References:

1. Sibai B, Dekker G, Kupferminc M. (2005) Pre-eclampsia. Lancet. 365, 785799

2. Maynard SE, Min JY, Merchan J, Lim KH, Li J, Mondal S, et al. Excess placental soluble fms-like tyrosine kinase 1 (sFlt1) may contribute to endothelial dysfunction, hypertension, and proteinuria in preeclampsia. J Clin Invest 2003; 111,649-58.

3. Lam $\mathrm{C}$, Lim $\mathrm{KH}$, Karumanchi SA. Circulating angiogenic factors in the pathogenesis and prediction of preeclampsia. Hypertension 2005; 46,1077-85.

4. Levine RJ, Karumanchi SA. Circulating angiogenic factors in preeclampsia. Clin Obstet Gynecol 2005; 48,372-86.

5. Levine RJ, Maynard SE, Qian C, Lim KH, England LJ, Yu KF et al. Circulating angiogenic factors and the risk of preeclampsia. N Engl J Med 2004; 350,672683.

6. Venkatesha S, Toporsian M, Lam C, Hanai JI, Mammoto T, Kim YM, et al. Soluble endoglin contributes to the pathogenesis of preeclampsia. Nat Med $2006 ; 12,642-9$.

7. Levine RJ, Lam C, Qian C, Yu KF, Maynard SE, Sachs BP, et al. Soluble endoglin and other circulating antiangiogenic factors in preeclampsia. $\mathrm{N}$ Engl J Med 2006; 355,992-1005.

8. Bills VL, Varet J, Millar A, Harper SJ, Soothill PW, Bates DO. Failure to upregulate VEGF165b in maternal plasma is a first trimester predictive marker for preeclampsia. Clin Sci (Lond). 2008 Oct 1. [Epub ahead of print] 
9. Vatten LJ, Eskild A, Nilsen TI, Jeansson S, Jenum PA, Staff AC. Changes in circulating level of angiogenic factors from the first to second trimester as predictors of preeclampsia. Am J Obstet Gynecol 2007; 196,239.e1-6.

10. Rana S, Karumanchi SA, Levine RJ, Venkatesha S, Rauh-Hain JA, Tamez H Thadhani R. Sequential changes in antiangiogenic factors in early pregnancy and risk of developing preeclampsia. Hypertension 2007; 50,35-36. 